\title{
Serum Uric Acid Levels in Different Phases of Acute Severe Manic and Depressed Patients
}

\author{
Mojtaba Keshavarz, ${ }^{1,}$ Fatemeh Khosravizadegan, ${ }^{2,3}$ and Alireza Bibak ${ }^{3}$ \\ ${ }^{1}$ Department of Pharmacology, School of Medicine, Bushehr University of Medical Sciences, Bushehr, IR Iran \\ ${ }^{2}$ Department of Psychiatry, School of Medicine, Bushehr University of Medical Sciences, Bushehr, IR Iran \\ ${ }^{3}$ Department of Psychiatry, Fatemeh Zahra Hospital, School of Medicine, Bushehr University of Medical Sciences, Bushehr, IR Iran \\ "Corresponding author: Mojtaba Keshavarz, Department of Pharmacology, School of Medicine, Bushehr University of Medical Sciences, Bushehr, IR Iran. Tel: +98-9177103353, \\ Fax: +98-7733320657, E-mail: mo.keshavarz@bpums.ac.ir
}

Received 2015 June 01; Revised 2015 November 17; Accepted 2015 November 24.

\begin{abstract}
Background: The level of serum uric acid, as an important endogenous antioxidant, may be correlated to the different phases of mood disorders.

Objectives: The aim of this study was to compare serum uric acid levels before and after treatment in patients with acute mania and depression.

Patients and Methods: We measured serum uric acid in 33 manic and 10 depressed hospitalized patients, both before and after treatment. Mood disorder was diagnosed according to the DSM-IV criteria. Manic or depressive scores were measured with the Young Mania Rating Scale or the Hamilton Depression Rating Scale, respectively. Uric acid levels were compared in the acute and remission phases of the mood disorder, and the relationship of uric acid levels with the onset of response was analyzed.

Results: Serum uric acid levels were increased after bipolar disorder treatment. Serum uric acid levels were increased after bipolar disorder treatment. Moreover, depressed patients with lower uric acid levels had a faster onset of response. The uric acid levels in the acute mania patients were higher than in the acute depression patients.

Conclusions: The remission phase, in comparison to acute mania or depression, had higher levels of uric acid. Moreover, lower serum uric acid may correlate to a faster response in depressed patients. These results may support the roles of the purinergic system and of oxidative stress in the treatment of mood disorders.
\end{abstract}

Keywords: Bipolar Disorder, Depressive Disorder, Remission Induction, Uric Acid

\section{Background}

Mood disorders are among the most debilitating psychiatric disorders, with increased mortality and morbidity in sufferers (1). Currently, trial and error is the basis for mood disorder pharmacotherapy mainly because of limitations in predicting treatment responses (2). Identifying objective biomarkers may lead to earlier diagnosis and treatment (3) by eliminating the subjective and symptomdependent treatments of mood disorders (4). In this regard, oxidative stress $(1,5)$ and the purinergic system (6) may offer promising clues, as they play important roles in the pathophysiology of mood disorders (7).

There are many pieces of evidence showing that oxidative stress is involved in the pathophysiology of mood disorders. It has been demonstrated that oxidative stress markers are increased in major depressive disorder (MDD) and bipolar disorder (BD) (8). Moreover, it was proposed that the therapeutic effects of mood stabilizers and antidepressant drugs might be at least partly related to the protection against oxidative stress damage in neuronal cells
$(8,9)$. Similarly, it has been suggested that the purinergic system might be involved in the pathophysiology of mood disorders $(10,11)$. Allopurinol's beneficial effects as a purinergic system modulator in the treatment of acute mania $(6,12)$ has encouraged many groups to consider uric acid an important biomarker for the monitoring of mood disorder treatments (13). In accordance with this idea, it was revealed that uric acid excretion is increased during the remission phase of mania (14). Moreover, plasma uric acid level alterations were positively correlated with Young Mania Rating Scale (YMRS) score changes (15). However, there are some discrepancies between studies with regard to the changes in uric acid levels during the different phases of mood disorders $(3,13,16)$.

\section{Objectives}

Currently, there are no diagnostic biomarkers available for mood disorders (17). It was proposed that uric acid, as an easily detectable marker (18), might be a predictor of different phases of mood disorders, and may be an index

Copyright (c) 2015, Tehran University of Medical Sciences. This is an open-access article distributed under the terms of the Creative Commons Attribution-NonCommercial 4.0 International License (http://creativecommons.org/licenses/by-nc/4.0/) which permits copy and redistribute the material just in noncommercial usages, provided the original work is properly cited. 
of the onset of action of mood stabilizers and antidepressant drugs. Considering the heterogeneity of mood disorders, the important drawback of previous studies may be related to comparisons of different patients in different phases of these disorders. Therefore, the aim of this study was to compare serum uric acid levels before and after treatment in the different phases of the mood disorder in patients with acute severe mania and depression.

\section{Patients and Methods}

\section{1. Subjects and Participants}

The study was a longitudinal study conducted over a nine-month period. Hospitalized patients, 35 with acute mania and 10 with acute depression, all between the ages of 18 and 65 years, were recruited at Bushehr Fatemeh Zahra Psychiatric Hospital in Iran. Age, educational level, marital status, and gender were recorded. Two patients were excluded during the course of the study (one for elevation of liver enzymes and one for a high blood urea nitrogen level).

Mood disorders (including BD and MDD) were diagnosed according to the DSM-IV criteria (19). The severity of mania was measured using the YMRS (15), while the Hamilton Rating Scale for Depression (HRSD) score was used to measure the severity of MDD (20). The manic patients were treated with mood-stabilizing agents (lithium and sodium valproate) and antipsychotics. The depressed patients were treated with antidepressants of different classes (mainly tricyclic antidepressants and serotonin reuptake inhibitors). The hospitalized patients were monitored by a psychiatrist every other day for one month, in order to note any changes in the mood condition.

The primary treatment response measures were the YMRS and HRSD scores. Subjects with YMRS of $\leq 7$ (6) and HRSD of $\leq 7$ (21) were considered to be remitted, and the first day of remission was considered to be at the onset of response. The exclusion criteria were disorders that may affect uric acid levels (gout, hypertension, and liver and kidney disorders), a history of allopurinol use, and alcohol or drug abuse.

After being informed of the details of the study, written consent forms were signed by the participants or their relatives. All procedures contributing to this work complied with the ethical standards of Bushehr University of Medical Science and the Iranian guidelines for the care of human subjects, which are in accordance with the provisions of the Declaration of Helsinki.

\section{2. Serum Uric Acid Measurements}

Using venipuncture, 5 -mL blood samples from the depressed patients and the manic patients were collected to measure serum uric acid. The serum uric acid level was determined via the spectrophotometric method using a commercially available enzymatic uric acid kit (Bionik Co., Canada) according to the manufacturer's instructions. Simultaneously, alanine transaminase (ALT), aspartate aminotransferase (AST), serum creatinine, blood urea nitrogen, and LDL and HDL cholesterol levels were measured with the relevant biochemical tests (Bionik Co., Canada) to monitor the liver, kidney, and lipid profiles of the subjects. All of the testing was carried out in the Khalije Phars Hospital biochemistry lab (Bushehr, Iran).

\section{3. Statistical Analysis}

After confirmation of the normal distribution of variables with the Shapiro-Wilk test, the paired t-test was used to compare uric acid mean values in the acute and remission phases of the mood disorder. The comparison of uric acid levels in the manic and depressed patients was performed using Student's t-test. The Pearson correlation was used to determine the relationship of uric acid level to the severity of mania or depression. Correspondingly, for assessing the uric acid level in relation to the onset of response, the Pearson correlation test was used. Additionally, Spearman's correlation was used to determine the relationship between uric acid and gender. To compare age, educational level, and marital status between the BD and MDD patients, the Mann-Whitney test was used. SPSS version 16.0 (SPSS Inc.) was used to perform the statistical analysis, and data were presented as mean \pm standard error of means (SEM). A P alue of $<0.05$ was considered significant.

\section{Results}

\subsection{Demographic Characteristics}

The subjects' demographic characteristics are shown in Table1. There were no differences in age, sex, educational level, and marital status between the BD and MDD patients.

\section{2. Uric Acid Levels and Clinical Features of Subjects}

The clinical features of the manic and depressed patients are summarized in Table 1 . The analysis revealed a correlation between uric acid level and the onset of response in the depressed patients $(\mathrm{r}=0.66, \mathrm{n}=10, \mathrm{P}=0.04)$. However, there was no correlation between the uric acid level and the onset of response in the acute manic patients $(\mathrm{r}=-0.1, \mathrm{n}=33, \mathrm{P}>0.05)$. When the manic and the depressed patients were considered together, there was no correlation between uric acid level and onset of response $(\mathrm{r}=0.14, \mathrm{n}=43, \mathrm{P}>0.05)$. In addition, there was no correlation between uric acid level and age or severity of mood 
Table 1. Clinical and Demographic Characteristics of the Subjects ${ }^{\mathrm{a}}$

\begin{tabular}{lcccc}
\hline & Age & Gender, Male & Mood Disorder Severity & Onset of Remission, days \\
\hline MDD & $39.90 \pm 3.34$ & 67 & $36.30 \pm 1.37$ & $8.00 \pm 1.10$ \\
BD & $36.50 \pm 1.88$ & 58 & $38.10 \pm 1.50$ & $16.66 \pm 1.28$ \\
Total & $37.28 \pm 1.63$ & 51 & NA & 10 \\
\hline
\end{tabular}

Abbreviations: BD, bipolar disorder; MDD, major depressive disorder; NA, not available.

${ }^{\mathrm{a}}$ Data are presented as mean \pm SD or No. \%.

${ }^{\mathrm{b}}$ Clinical features and demographic characteristics of 33 manic and 10 depressed patients were registered, and mood disorder severity was measured with the YMRS or the HDRS for mania or depression, respectively.

disorder $(\mathrm{P}>0.05)$. However, there was a correlation between uric acid level and $\operatorname{sex}(\mathrm{r}=0.34, \mathrm{n}=43, \mathrm{P}<0.05)$.

When compared before and after treatment, BD patients in the remission phase had significantly higher levels of serum uric acid ( $t=-2.50,95 \%$ CI -0.97, -0.10) (Figure 1). In depressed patients, serum uric acid levels were higher in the remission phase compared to the acute phase. However, there was no significant difference between the acute and remission phases in the depressed patients $(\mathrm{t}=-1.17$, 95\% CI -1.11, 0.35) (Figure 1). In addition, when compared before treatment, uric acid levels were higher in the acute mania patients than in the acute depression patients (4.66 \pm 0.21 and $3.69 \pm 0.35$, respectively; $\mathrm{t}=-2.25$, 95\% CI -1.84 , 0.10 ) (Figure 1). Similarly, in the remission period, uric acid levels in the acute mania patients were higher than in the acute depression patients $(5.20 \pm 0.24$ and $4.07 \pm 0.49$, respectively; $\mathrm{t}=-2.16,95 \% \mathrm{CI}-2.18,0.07)$.

Figure 1. Serum Uric Acid Levels in Patients With Acute Severe Mania and Depression

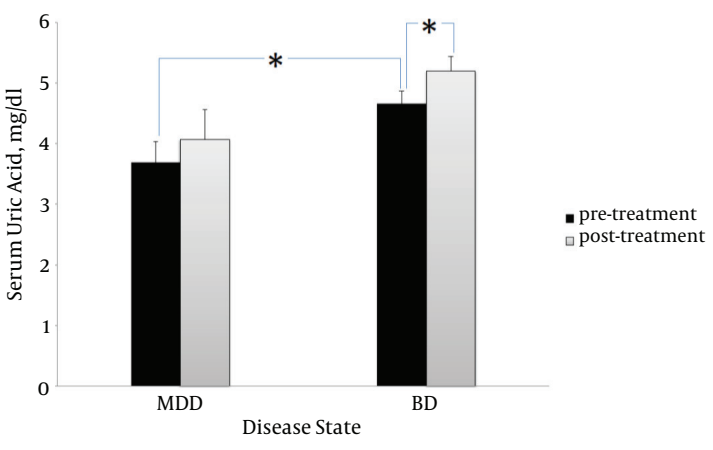

The mean serum uric acid levels in the acute and remission phases of major depression patients $(n=10)$ and manic patients $(n=33)$ were investigated before and after treatment with mood-stabilizing agents, antidepressants, and antipsychotics. The paired t-test was used to compare before and after treatment, and Student's ttest was performed for the comparison between manic and depressed patients. ${ }^{*} \mathrm{P}<$ 0.05. MD: major depression, BD: bipolar disorder.

\section{Discussion}

The present study performed longitudinal measurements of serum uric acid to permit the evaluation of this biomarker in different phases of the mood disorder. The results showed that the remission phase, in comparison to the acute manic phase, involved higher levels of uric acid in the acute BD patients. Moreover, the uric acid levels during the remission phase of MDD were higher compared to the before-treatment phase; however, this difference was not significant. There are very limited studies that compare uric acid levels before and after treatment in BD and MDD patients. In agreement with our results, chronic antidepressant treatment increased serum uric acid levels in depressed patients (18). In contrast to our findings, it was reported that uric acid levels during acute manic episodes were higher than during the remission phase (13). In contrast to De Berardis et al., we assessed the serum uric acid levels in the acute and remission phases of the same patients. Although they were matched for certain factors such as age and smoking, it is almost impossible to delete all confounding factors in different individuals with complicated conditions such as mood disorders.

The exact reason for rising uric acid levels in the process of acute mania treatment is not completely clear. However, this might be at least partly related to the antipsychotics and mood-stabilizing agents used in these patients. In our study, all manic patients received antipsychotic drugs as adjunctive treatment along with moodstabilizing drugs. Some reports have shown that neuroleptics increase uric acid levels in schizophrenia (22). Moreover, it has been shown that valproate can increase uric acid levels (23). Therefore, uric acid changes might be related to the effects of antipsychotic drugs and valproate, rather than to treatment effects. It is noteworthy that patients were under treatment with drugs from different classes, and the reported effects were possibly related to all of the drugs used. Thus, it was impossible to distinguish between drug effects and treatment responses in these patients. We monitored the manic patients for a short period 
of time, and these effects only involved acute alterations in the uric acid levels of these patients.

Uric acid increment in the remission phase of BD and MDD may support the role of uric acid and oxidative stress in the pathology and treatment of mood disorders. Uric acid is a natural antioxidant, with high levels of free-radical scavenging activity in the blood (24) and brain (25). It has been suggested that low uric acid levels are associated with the development and progression of a variety of central nervous system (CNS) diseases (26). In parallel, it was proposed that the reduced uric acid level in the acute phase of mood disorders might reflect the reduction of natural antioxidant (3). In our study, we observed lower levels of serum uric acid in the acute phase of the mood disorder. This observation may be due to the increased utilization of this substance for scavenging free radicals in the brain (27). In line with this idea, it is acknowledged that BD and MDD are associated with increased free-radical production and decreased antioxidant defenses $(1,27)$. Moreover, in vitro and in vivo studies have shown that mood-stabilizing agents augment antioxidant defenses $(27,28)$. Accordingly, it can be assumed that the acute phase of the mood disorder is accompanied by a reduced level of uric acid as an important antioxidant. Moreover, pharmacotherapy may increase uric acid to protect neuronal cells against oxidative stress damage in mood disorders. In this regard, lower uric acid has been associated with some neurodegenerative disorders, such as Parkinson's and Alzheimer's disease $(29,30)$. After considering uric acid's ability to suppress CNS damage and neuronal death (31), it is possible to suggest that rising uric acid in the remission period may help to prevent neuronal damage in mood disorders.

Uric acid levels in the BD patients were higher in comparison to the depressed patients. In agreement with this study, it has been demonstrated that uric acid levels in depressed patients were lower than in other mental disorders, including BD, in hospitalized patients (18). In addition, another study has shown that uric acid levels during the remission phase of manic patients were higher than in depressed patients and healthy volunteers (32). Moreover, it was shown that BD patients were more prone to suffering from gout when compared with healthy controls (33). In this regard, it was proposed that a high serum uric acid level might be an indicator for differentiating between mania and depression (32).

At present, the basis for mood disorder treatment mainly relies on trial and error, and there is no objective parameter for predicting responses in these disorders. Moreover, there is a lag time after treatment initiation with antidepressant drugs that may be very dangerous in depressed patients. For instance, at least four weeks are necessary to reach remission after initial selective serotonin reuptake inhibitor (SSRI) antidepressant treatment (34). Therefore, it is very important to predict the treatment outcome and the onset of response in mood disorders in order to reduce the patient's suffering (35). This study, for the first time, showed that depressed patients with lower levels of uric acid had more rapid onset of response. This may be helpful in predicting patients' responses to antidepressant treatments. Accordingly, uric acid, as an indicator of the purinergic and oxidative stress systems, may be a new target for the development of drugs that could accelerate treatment responses in mood disorders.

There was no correlation between uric acid levels and severity of mood disorders in both the manic and the depressed patients. In agreement with our results, it was revealed that uric acid levels were not significantly correlated with the severity and duration of MDD (18). As an explanation, the lack of association between uric acid and mood severity in our study may be related to the homogeneity of the patients that we recruited. Consequently, if patients with disorders of lower severity were included in the study, a relationship between uric acid levels and severity of mood disorders might be demonstrated.

Our study had some limitations. It is important to note that this study was conducted using a small number of patients with severe acute mania and depression. Studies with larger sample sizes seem necessary. Moreover, most of the patients were not drug-naive before participation in the study.

In conclusion, this study presents further proof of the involvement of uric acid in the pathogenesis and treatment of mood disorders. Rising uric acid during treatment for acute mania and depression may be an indicator of an antioxidant defense increment in the course of the treatment. Therefore, it can be proposed that a uric acid increment during the treatment course for acute mania may be a marker for monitoring successful treatment of this disorder. Moreover, lower uric acid levels may be a predicting factor for accelerated onset of response to antidepressants.

\section{Acknowledgments}

We greatly appreciate the Deputy for Research at Bushehr University of Medical Sciences for providing the budget and helping to carry out this research. The authors declare no conflict of interest.

\section{Footnotes}

Authors' Contribution: Mojtaba Keshavarz, developed the proposal, managed the study design, interpreted the 
data, and drafted and revised the final version of the paper; Fatemeh Khosravizadegan, helped with the study design and acquisition of data, and helped draft and revise the article; Alireza Bibak, helped with the study design, acquisition of data, drafting and revision of the data, and final approval of the article, Proposal introduction, managing the study design, interpretation of data, drafting and revision of the data, and final approval of the article.

Funding/Support: This study was financially supported by the Deputy for Research at Bushehr University of Medical Sciences.

\section{References}

1. Andreazza AC, Kauer-Sant'anna M, Frey BN, Bond DJ, Kapczinski F, Young LT, et al. Oxidative stress markers in bipolar disorder: a meta-analysis. J Affect Disord. 2008;111(2-3):135-44. doi: 10.1016/j.jad.2008.04.013. [PubMed: 18539338].

2. Kowatch RA, Fristad M, Birmaher B, Wagner KD, Findling RL, Hellander M, et al. Treatment guidelines for children and adolescents with bipolar disorder.J Am Acad Child Adolesc Psychiatry. 2005;44(3):213-35. [PubMed: 15725966]

3. Chaudhari K, Khanzode S, Khanzode S, Dakhale G, Saoji A, Sarode S. Clinical correlation of alteration of endogenous antioxidanturic acid level in major depressive disorder. Indian J Clin Biochem. 2010;25(1):77-81. doi: 10.1007/s12291-010-0016-z. [PubMed: 23105889].

4. Le-Niculescu H, McFarland MJ, Ogden CA, Balaraman Y, Patel S, Tan J, et al. Phenomic, convergent functional genomic, and biomarker studies in a stress-reactive genetic animal model of bipolar disorder and co-morbid alcoholism. Am J Med Genet B Neuropsychiatr Genet. 2008;147B(2):134-66. doi: 10.1002/ajmg.b.30707. [PubMed: 18247375].

5. Teixeira AL, Barbosa IG, Machado-Vieira R, Rizzo LB, Wieck A, Bauer ME. Novel biomarkers for bipolar disorder. Expert Opin Med Diagn. 2013;7(2):147-59. doi: 10.1517/17530059.2013.734807. [PubMed: 23530885].

6. Machado-Vieira R, Soares JC, Lara DR, Luckenbaugh DA, Busnello JV, Marca G, et al. A double-blind, randomized, placebo-controlled 4week study on the efficacy and safety of the purinergic agents allopurinol and dipyridamole adjunctive to lithium in acute bipolar mania. J Clin Psychiatry. 2008;69(8):1237-45. [PubMed: 18681754]

7. Rosa AR, Singh N, Whitaker E, de Brito M, Lewis AM, Vieta E. Altered plasma glutathione levels in bipolar disorder indicates higher oxidative stress; a possible risk factor for illness onset despite normal brain-derived neurotrophic factor (BDNF) levels. Psychol Med. 2014;27:1-10. doi: 10.1017/s0033291714000014.

8. Ng F, Berk M, Dean O, Bush AI. Oxidative stress in psychiatric disorders: evidence base and therapeutic implications. Int J Neuropsychopharmacol. 2008;11(6):851-76. doi: 10.1017/S1461145707008401. [PubMed: 18205981].

9. Berk M, Kapczinski F, Andreazza AC, Dean OM, Giorlando F, Maes M, et al. Pathways underlying neuroprogression in bipolar disorder: focus on inflammation, oxidative stress and neurotrophic factors. Neurosci Biobehav Rev. 2011;35(3):804-17. doi: 10.1016/j.neubiorev.2010.10.001. [PubMed: 20934453].

10. Barden N, Harvey M, Gagne B, Shink E, Tremblay M, Raymond C, et al. Analysis of single nucleotide polymorphisms in genes in the chromosome $12 \mathrm{Q} 24.31$ region points to $\mathrm{P} 2 \mathrm{RX} 7$ as a susceptibility gene to bipolar affective disorder. Am J Med Genet B Neuropsychiatr Genet. 2006;141B(4):374-82. doi:10.1002/ajmg.b.30303. [PubMed:16673375].

11. Sperlagh B, Csolle C, Ando RD, Goloncser F, Kittel A, Baranyi M. The role of purinergic signaling in depressive disorders. Neuropsychopharmacol Hung. 2012;14(4):231-8. [PubMed: 23269209].
12. Akhondzadeh S, Milajerdi MR, Amini H, Tehrani-Doost M. Allopurinol as an adjunct to lithium and haloperidol for treatment of patients with acute mania: a double-blind, randomized, placebocontrolled trial. Bipolar Disord. 2006;8(5 Pt 1):485-9. doi: 10.1111/j.13995618.2006.00363.x. [PubMed: 17042886].

13. De Berardis D, Conti CM, Campanella D, Carano A, Di Giuseppe B, Valchera A, et al. Evaluation of plasma antioxidant levels during different phases of illness in adult patients with bipolar disorder. J Biol Regul Homeost Agents. 2008;22(3):195-200. [PubMed: 18842173].

14. Anumonye A, Reading HW, Knight F, Ashcroft GW. Uric-acid metabolism in manic-depressive illness and during lithium therapy. Lancet. 1968;1(7555):1290-3. [PubMed: 4172145].

15. Young RC, Biggs JT, Ziegler VE, Meyer DA. A rating scale for mania: reliability, validity and sensitivity. Br J Psychiatry. 1978;133:429-35. [PubMed: 728692].

16. Lee Y, Han D, Noh D, Kim S, Na C. Correlation between clinical symptoms, serum uric acid level, and beta wave of EEG in patients with bipolar disorder. Eur Neuropsychopharmacol. 2010;20:S408.

17. Hashimoto K. Brain-derived neurotrophic factor as a biomarker for mood disorders: an historical overview and future directions. Psychiatry Clin Neurosci. 2010;64(4):341-57. doi: 10.1111/j.14401819.2010.02113.x. [PubMed: 20653908].

18. Wen S, Cheng M, Wang H, Yue J, Wang H, Li G, et al. Serum uric acid levels and the clinical characteristics of depression. Clin Biochem. 2012;45(1-2):49-53. doi: 10.1016/j.clinbiochem.2011.10.010. [PubMed: 22040815].

19. American Psychiatric Association . diagnostic criteria from dsM-iV-tr. Arlington: American Psychiatric Pub; 2000.

20. Hamilton M. A rating scale for depression.J Neurol Neurosurg Psychiatry. 1960;23:56-62. [PubMed: 14399272].

21. McIntyre RS, Mancini DA, McCann S, Srinivasan J, Sagman D, Kennedy $\mathrm{SH}$. Topiramate versus bupropion SR when added to mood stabilizer therapy for the depressive phase of bipolar disorder: a preliminary single-blind study. Bipolar Disord. 2002;4(3):207-13. [PubMed: 12180276].

22. Reddy R, Keshavan M, Yao JK. Reduced plasma antioxidants in firstepisode patients with schizophrenia. Schizophr Res. 2003;62(3):20512. [PubMed: 12837516].

23. Belcastro V, D'Egidio C, Striano P, Verrotti A. Metabolic and endocrine effects of valproic acid chronic treatment. Epilepsy Res. 2013;107(1-2):18. doi:10.1016/j.eplepsyres.2013.08.016. [PubMed: 24076030].

24. Stinefelt B, Leonard SS, Blemings KP, Shi X, Klandorf H. Free radical scavenging, DNA protection, and inhibition of lipid peroxidation mediated by uric acid. Ann Clin Lab Sci. 2005;35(1):37-45. [PubMed: 15830708].

25. Bowman GL, Shannon J, Frei B, Kaye JA, Quinn JF. Uric acid as a CNS antioxidant. J Alzheimers Dis. 2010;19(4):1331-6. doi: 10.3233/JAD-20101330. [PubMed: 20061611].

26. Kutzing MK, Firestein BL. Altered uric acid levels and disease states. J Pharmacol Exp Ther. 2008;324(1):1-7. doi: 10.1124/jpet.107.129031. [PubMed: 17890445].

27. Ozcan ME, Gulec M, Ozerol E, Polat R, Akyol O. Antioxidant enzyme activities and oxidative stress in affective disorders. Int Clin Psychopharmacol. 2004;19(2):89-95. [PubMed: 15076017].

28. Shao L, Young LT, Wang JF. Chronic treatment with mood stabilizers lithium and valproate prevents excitotoxicity by inhibiting oxidative stress in rat cerebral cortical cells. Biol Psychiatry. 2005;58(11):879-84. doi: 10.1016/j.biopsych.2005.04.052. [PubMed: 16005436].

29. Kim TS, Pae CU, Yoon SJ, Jang WY, Lee NJ, Kim JJ, et al. Decreased plasma antioxidants in patients with Alzheimer's disease. Int J Geriatr Psychiatry. 2006;21(4):344-8. doi: 10.1002/gps.1469. [PubMed: 16534775].

30. de Lau LM, Koudstaal PJ, Hofman A, Breteler MM. Serum uric acid levels and the risk of Parkinson disease. Ann Neurol. 2005;58(5):797-800. doi: 10.1002/ana.20663. [PubMed:16240356]. 
31. Yu ZF, Bruce-Keller AJ, Goodman Y, Mattson MP. Uric acid protects neurons against excitotoxic and metabolic insults in cell culture, and against focal ischemic brain injury in vivo. J Neurosci Res. 1998;53(5):613-25. [PubMed: 9726432].

32. Kesebir S, Tatlidil Yaylaci E, Suner O, Gultekin BK. Uric acid levels may be a biological marker for the differentiation of unipolar and bipolar disorder: the role of affective temperament. J Affect Disord. 2014;165:131-4. doi: 10.1016/j.jad.2014.04.053. [PubMed: 24882190].

33. Chung $\mathrm{KH}$, Huang CC, Lin HC. Increased risk of gout among patients with bipolar disorder: a nationwide population-based study. Psy- chiatry Res. 2010;180(2-3):147-50. doi: 10.1016/j.psychres.2009.07.012. [PubMed: 20483460].

34. Trivedi MH, Fava M, Wisniewski SR, Thase ME, Quitkin F, Warden D, et al. Medication augmentation after the failure of SSRIs for depression. N Engl J Med. 2006;354(12):1243-52. doi: 10.1056/NEJMoa052964. [PubMed: 16554526].

35. Insel TR, Scolnick EM. Cure therapeutics and strategic preven tion: raising the bar for mental health research. Mol Psychiatry. 2006;11(1):11-7. doi: 10.1038/sj.mp.4001777. [PubMed: 16355250]. 